\title{
Potocki-Lupski Syndrome: Holistic Dental Management at Primary Healthcare - Qatar
}

\author{
Abdul Morawala, Makhtoum Abdul Azeez, Maha Saif AlMesallam, Asmaa Alkhtib, Ahmed A. \\ Elbayoumi, Shweta Jajoo
}

\section{ABSTRACT}

The holistic dentistry is one of the oldest biological forms of dentistry which highlights the importance of oral health and its impact on the overall health of the patient. It roots deeply in prevention of causative factor of diseases instead of treating it using potentially toxic materials. Holistic approach is helpful in children with congenital developmental disorders as such patients have restrictions that are physical or mental which leads to deterioration in self-care, and oral care state. Potocki Lupski syndrome is one such syndrome where the child presents feature of infantile hypotonia, sleep disorder, congenital anomalies of the cardiovascular system, poor oral motor skills and crowded dentition that leads to failure to thrive. In this report, we present a case of a 4-year-old child with Potocki Lupski syndrome who was intervened though a holistic dentistry using a multidisciplinary approach.

Keywords: Behavioral disturbances, cognitive delay, failure to thrive, holistic dentistry, Potocki-Lupski syndrome, preventive medicine.
Published Online: January 25, 2022

ISSN: $2684-4443$

DOI: $10.24018 /$ ejdent.2022.3.1.85

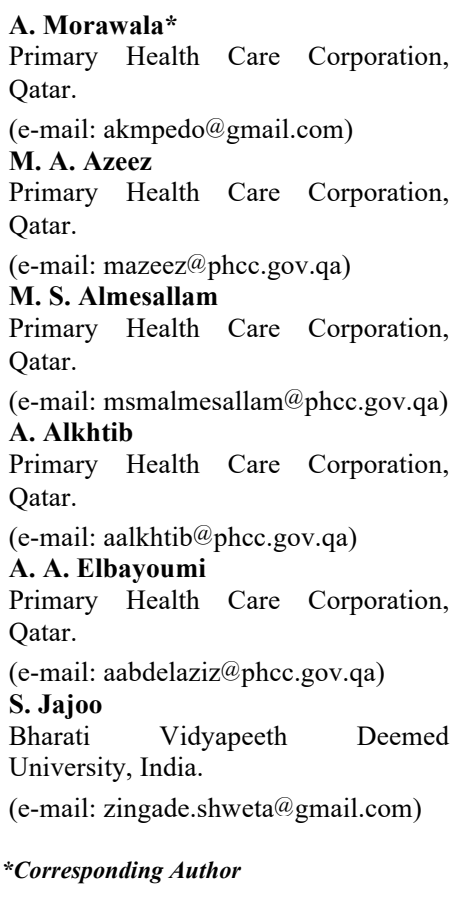

\section{INTRODUCTION}

Holistic healthcare model promotes a healthy well-being of an individual through adequate nourishment, removal of toxic products while keeping in harmony with the individuals physical, mental and energy levels. It is well known that oral health is overall health, as the oral cavity is not just merely for mastication and speech, but it presents a reflection of the individuals over-all health. A concept which forms the framework of Holistic dentistry [1]. As the dynamic activity of the oral cavity is ultimately responsible for the skeletal mechanism and nutritional biochemistry.

Holistic approach is like traditional dentistry except that a more biological and a preventive approach is often sought instead of applying treatment with potentially harmful substances and dates back to 1800 s when it was found that amalgam is a potentially toxic compound in restorative fillings [2]. It employs the usage of natural products that are highly biocompatible and rooted in prevention rather than curing problems as and when it arises. In children with syndromes or those who are specially challenged, Prevention forms the best method of treatment, as eliminating the root causes of disease likely reduces the chances of it ever occurring.

Potocki-Lupski syndrome (PTLS), named after Lorraine Potocki and James R. Lupski was first described by [3], [4] in 1996. It occurs due to a microduplication of fragment of chromosome localized to the RAI1 (retinoic acid 1) gene harboring the chromosome $17 \mathrm{p} 11.2$ and this gene is responsible for the clinical manifestations of this disorder. It involves approximately 1:25.000 people [5].

It is an autosomal dominant, cognitive, behavioral developmental disorder characterized by symptoms ranging from mild to severe including delayed milestones, hypotonia, failure to thrive, mental retardation, pervasive developmental disorders, congenital and cardiovascular anomalies [6], [7]. Physical features are diagnosed at infancy while the cognitive delay and psychological manifestations are diagnosed during later stages. Children with PTLS have facial dysmorphic with triangular facies, smooth philtrum, high-arched palate, frontal 
bossing, mandibular and maxillary hypoplasia, submucous cleft palate and bifid uvula [7] along with delayed speech is found to be prevalent in all forms of PTLS [7] They are usually having short stature due to growth hormone deficiency and dental abnormalities such crowded dentition with dental caries due to poor development of motor skills.

International Standard Cytogenomic Array (ISCA) Consortium stated that chromosomal microarray should be performed as the first line in cytogenetic diagnostic test on patients with developmental delay/intellectual disability, autism disorders and multiple congenital anomalies as the chromosomal array has a higher sensitivity compared to the conventional G- banding karyotyping [5].

Poor oral motor skills and hypotonia in such patients lead to failure to thrive. A comprehensive medical history recording is important for the evaluation of such patients. Preventive measures are given priority as addressing the problems in the future necessitates treatment using potentially toxic products that would otherwise affect their overall health. Here we present a case oral rehabilitation using a holistic approach on 4-year-old boy with PTLS syndrome and emphasize on their importance.

\section{CASE REPORT}

The authors of the accepted manuscripts will be given a copyright form and the form should accompany your final submission.

A 4-year-old boy, first child of a non-consanguineous marriage presented to the hospital with problems of delayed speech, hypotonia, recurrent ear infection, and fluid behind ear drums. Post-natal history was taken. APGAR score was 7 and 8 at 1 and 5 min respectively. Which indicates he was new-born; he was in good condition and only required routine post-partum care. He had started walking at 2 years of age and understands commands but could pronounce a few words. History of Cryptorchidism was noted and had been operated on.

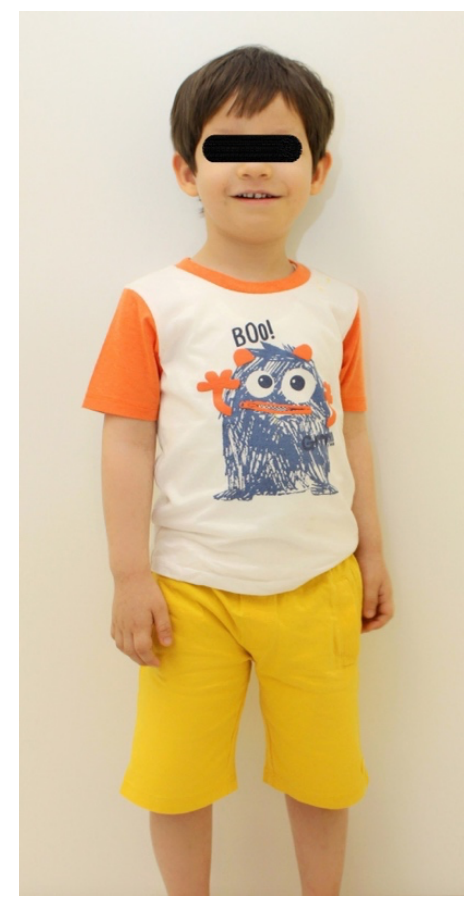

Fig. 1. Clinical photograph of the child.
On clinical examination, patient appeared to be happy. Some extraoral findings revealed spare outer third of eyebrows, everted outer end of the lower eyelid and Hypotonia of muscle (Fig. 1). Oral examination revealed all primary teeth were present with white spot carious lesions are seen. The first visit was done in January 2019 (Fig. 2 and 3).

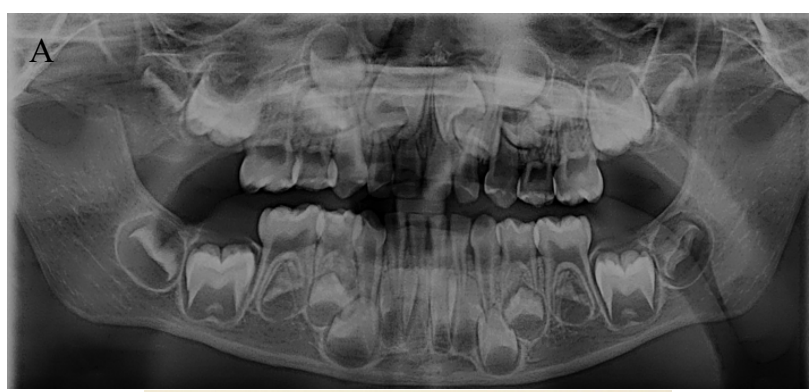

B

B

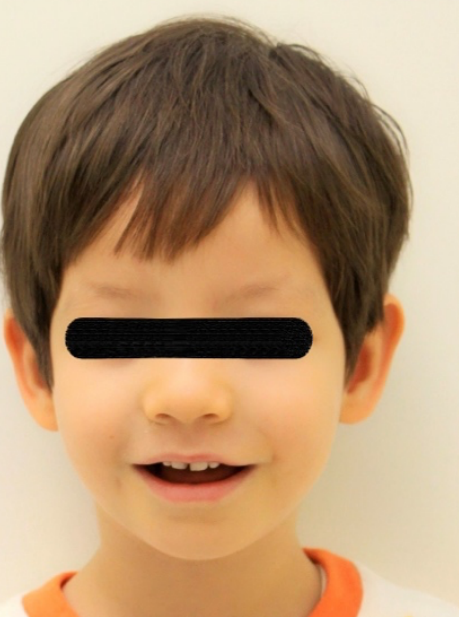

Fig. 2. First visit Panoramic radiograph.

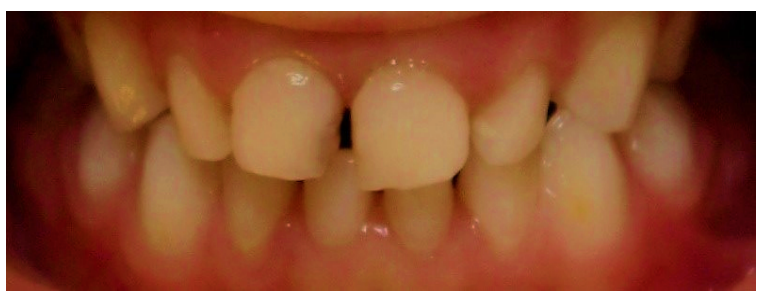

Fig. 3. First visit oral photograph.

In the first phase of treatment plan, Preventive measures were undertaken namely, Pit and fissure sealants using fluoride was done. Considering the history of the patient, along with the presenting features, a whole genome wide oligonucleotide array-based comparative genomic hybridization analysis was performed with use of Human Genome CGH microarray Kit. The results showed that the patient carried an interstitial gain of $3.8 \mathrm{Mb}$ within cytogenic band 17p 11.2. The duplication segment includes RA11 gene, thereby confirming the diagnosis of Potocki Lupski syndrome. The patient was followed up for every 6 months for three years for continuous fluoride application. Genetic counselling was offered for the parents in case of future pregnancies.

After 3 years, the oral health of the child appeared to be in a sound state. A panoramic radiograph was taken to evaluate the eruption status of the permanent teeth (Fig. 4) which revealed normal tooth buds and erupting permanent teeth. 


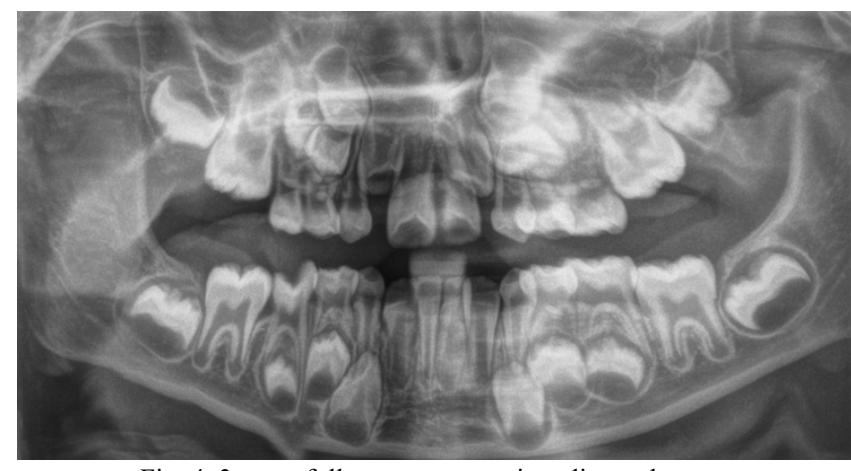

Fig. 4. 3 years follow up panoramic radiograph.

\section{DiscUSSION}

Traditional medicine and dentistry are limited to treating a problem related with a specific system, In the recent years, people have become aware and are constantly seeking more natural, biocompatible treatment that produces minimal adverse effects, one among such discipline of medicine is the holistic medicine, or holistic dentistry [6]. Holistic dentistry is concerned with the effects of oral health on the general health of the patient. This approach interconnects oral health with the rest of the body; including every single body organ and system as it not only it treats the problems associated with the teeth but also acknowledges the mind, body, and spirit of the patient [7].

Using the basic principles to holistic dentistry, oral probiotics were also prescribed to have a positive shift of microbiota [8]. The pits and fissures of the child's tooth under preventive care was sealed using fluoride, thereby avoiding any toxins from other dental materials in the future. In each visit, care was taken to ensure the prevention of dental malocclusion, proper oral hygiene practices were instructed for the children as well as the parent to follow to prevent any gum disease at the biological basis [9], [10].

Holistic dentistry and PTLS syndrome necessitate an interdisciplinary approach, A nutritional counsellor had educated and advised the parents about the changes needed to be implemented in child's diet to provide a healthy, more immune, and internally strong body, thereby preventing or reversal degenerative dental disease.

Children with Potocki Lupski syndrome present with delayed development, cognitive skills behavioral impairments including disturbed sleep-wake cycle, hearing problems, dental and skeletal abnormalities, and abnormal kidney development and function [10]. Cardiovascular anomalies in $40 \%$ of children with PTLS [11]. Cognitive features include delayed developmental milestones, intellectual disability, behavioral disorders, withdrawal, attention deficit hyperactivity disorder ADHD, autism spectrum disorders-ASD), motor clumsiness/coordination impairment [12].

In our case, the patient showed a milder phenotype of PTLS with minimal facial dysmorphism, mild intellectual disabilities, and slight language developmental delay without any internal systemic organ involvement. There were no other neurological or autistic features. Children with Potocki Lupski syndrome have delayed developmental and gross motor skills such as sitting, standing, and walking. None of which were observed in our patient.

There is no specific treatment for children with PTLS. In the present case, multidisciplinary approach was used which involved sessions with genetic consultant, Pedodontist, Pediatrician, speech therapist, audiologist, nurses, pathologists, radiologists, physiotherapists etc.

Dietary changes were made which included addition of health fibrous foods, restriction on refined sugar products with emphasis on the role of carbohydrate on dental caries was undertaken. As doing this reduces the likelihood of caries susceptibility, as carbohydrates are well known to increase the risk for dental caries [13]. The need to treat future dental caries/ issues with toxic contents from amalgam and Composite resin filling was eliminated in our patients by administering fluoride pit and fissure sealant. A careful monitoring of the patient for any dental crowding/malocclusion throughout the follow up visits were exercised. Proper oral hygiene instructions were given to reduce the prevalence of gum disease.

\section{CONCLUSION}

Children with PTLS present with high risk for developmental and neuromuscular disorder characterized by lack of nutrition due to poor dentition, oral hygiene, and oropharyngeal dysphagia. A holistic approach with a special emphasis on prevention is resourceful for the parents as well as children.

\section{ACKNOWLEDGEMENTS}

The author would like to thank Primary Healthcare Corporation - Qatar \& Dr Kimaya Kade BVDC - Pune for their support.

\section{REFERENCES}

[1] Nadine A. Holistic Oral Care: Avoid the Dentist Without Ignoring Your Teeth. 2015.

[2] Brownn A, Phelan MC, Patil S, Crawford E, Rogers RC, Schwartz C. Two patients with duplication of $17 \mathrm{p} 11.2$ : the reciprocal of the SmithMagenis syndrome deletion? Am J Med Genet. 1996; 63: 373-7.

[3] Dalai DR, Bhaskar DJ, Agali CR, Punya H. Holistics: A New Approach in Dentistry. Int J Adv Sci. 2014: 1(3): 323.

[4] Potocki L, Bi W, Treadwell-Deering D, Carvalho CM, Eifert A, Friedman EM, et al. Characterization of Potocki-Lupski syndrome (dup (17) (p11.2p112)) and delineation of a dosage-sensitive critical interval that can convey an autism phenotype. Am J Hum Genet. 2007; 80: 63349.

[5] Potocki L, Neira-Fresneda J, Yuan B. Potockilupski syndrome. 2017.

[6] Liu P, Lacaria M, Zhang F, Withers M, Hastings PJ, Lupski JR. Frequency of nonallelic homologous recombination is correlated with length of homology: evidence that ectopic synapsis precedes ectopic crossing-over. Am J Hum Genet. 2011; 89: 580-8.

[7] Shchelochkov OA, Cheung SW, Lupski JR. Genomic and clinical characteristics of microduplications in chromosome 17. Am J Med Genet. 2010; 152A: 1101-1110.

[8] Ciaccio C, Pantaleoni C, Milani D, Alfei E, Sciacca FL, Canafoglia L, et al. Neurological phenotype of Potocki-Lupski syndrome. Am J Med Genet. 2020; 182: 2317-24.

[9] Potockii L, Chen K-S, Park S-S, Osterholm DE, Withers MA, Kimonis $\mathrm{V}$, et al. Molecular mechanism for duplication $17 \mathrm{p} 11.2$ - the homologous recombination reciprocal of the Smith-Magenis microdeletion. Nature Genet. 2000; 24: 84-87. 
[10] Neira-Fresneda J, Potocki L. Neurodevelopmental disorders associated with abnormal gene dosage: Smith-Magenis and Potocki-Lupski Syndromes. J Pediatr Genet. 2015; 4: 159-67.

[11] Stankiewicz P, Lupski JR. Structural variation in the human genome and its role in disease. Ann Rev Med. 2010; 61: 437-55.

[12] Goh ES-Y, Perez IC, Canales CP, Ruiz P, Agatep R, Yoon G, et al. Definition of a critical genetic interval related to kidney abnormalities in the Potocki-Lupski syndrome. Am J Med Genet. 2012; 158A: 157988.

[13] Jefferies JL, Pignatelli RH, Martinez HR, Robbins-Furman PJ, Liu P, $\mathrm{Gu} \mathrm{W}$, et al. Cardiovascular findings in duplication $17 \mathrm{p} 11.2$ syndromes. Genet Med. 2012; 14: 90-4.

[14] Treadwell-Deering DE, Powell MP, Potocki L. Cognitive and behavioral characterization of the Potocki-Lupski syndrome (duplication 17p11.2). J Dev Behav Pediatr. 2010; 31: 137-43.

[15] Tungare S, Paranjpe AG. Diet and Nutrition to Prevent Dental Problems. [Internet] 2021 [cited 2021 Aug 11]. Available from: https://www.ncbi.nlm.nih.gov/books/NBK534248/ 